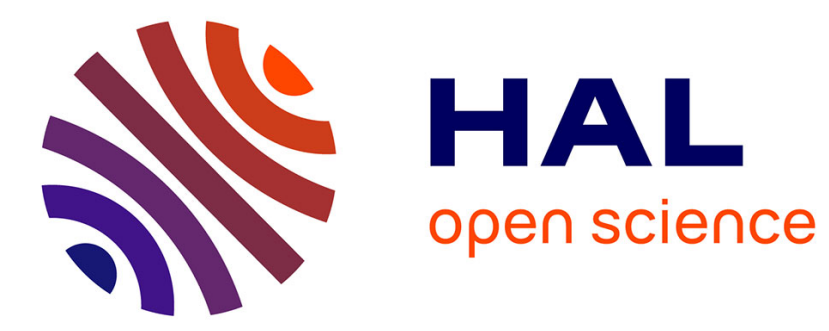

\title{
Global dynamics of the chemostat with overflow metabolism
}

\author{
Carlos Martínez, Jean-Luc Gouzé
}

\section{To cite this version:}

Carlos Martínez, Jean-Luc Gouzé. Global dynamics of the chemostat with overflow metabolism. Journal of Mathematical Biology, 2021, 82 (3), 10.1007/s00285-021-01566-6 . hal-03130387

\section{HAL Id: hal-03130387 https://hal.inria.fr/hal-03130387}

Submitted on 3 Feb 2021

HAL is a multi-disciplinary open access archive for the deposit and dissemination of scientific research documents, whether they are published or not. The documents may come from teaching and research institutions in France or abroad, or from public or private research centers.
L'archive ouverte pluridisciplinaire HAL, est destinée au dépôt et à la diffusion de documents scientifiques de niveau recherche, publiés ou non, émanant des établissements d'enseignement et de recherche français ou étrangers, des laboratoires publics ou privés. 


\title{
Global dynamics of the chemostat with overflow metabolism
}

\author{
Carlos Martínez * \\ Jean-Luc Gouzé ${ }^{\dagger}$ \\ Université Côte d'Azur, Inria, INRAE, CNRS, Sorbonne Université \\ Biocore team, Sophia Antipolis, France
}

February 3, 2021

\begin{abstract}
We analyze the asymptotic behavior of the periodically forced light limited Droop model, representing microalgae growth. We consider general monotone growth and uptake rate functions. Based on a conservation principle, we reduce the model to a limiting planar periodic system of differential equations. The reduced system generates a monotone dynamical system. Combining this fact with results on periodic Kolmogorov equations, we find conditions such that any solution of the reduced model approaches to a positive periodic solution. Under these conditions, if the reduced system admits only one positive periodic solution, using the theory of asymptotically periodic semiflows, we extend the results on the limiting system to the original model. Finally, based on results of monotone sub-homogeneous dynamical systems, we give conditions to determine the uniqueness of positive periodic solutions.
\end{abstract}

\section{Introduction}

Escherichia coli $(E$. coli) is a bacterium that is naturally found in the intestine of humans and other mammals. This bacterium has been a preferred choice for large-scale production of recombinant proteins ${ }^{1}$ such as insulin, GFP (green fluorescent protein), or the human growth hormone [3] [17]. For high density cultivation of $E$. coli, glucose is generally the preferred and most common carbon and energy source [6], since this is inexpensive and readily utilizable. To harvest energy from glucose, E. coli combines two different metabolic strategies, aerobic respiration, which needs oxygen, and fermentation, which does not need oxygen [12]. Respiration is more energy-efficient than fermentation, nevertheless, in

\footnotetext{
*carlos.martinez@inria.fr

$\dagger$ jean-luc.gouze@inria.fr

${ }^{1}$ Recombinant proteins are proteins that are artificially made through the recombinant DNA technology.
} 
fast growing cells, some energy is also obtained by fermentation. This seemingly wasteful strategy in which cells use fermentation instead of respiration, even in the presence of oxygen, is known as overflow metabolism [4]. This phenomenon is not only limited to E. coli, but to a diverse range of microorganisms [30]. For example, in yeasts, overflow metabolism is known as Crabtree effect [8], and in cancer cells it is known as Warburg effect [18].

Overflow metabolism results in the secretion of fermentation by-products, such as acetate in E. coli cultures or ethanol in yeast cultures, which accumulation can have an inhibitory effect on cells growth. For example, glucose uptake is inhibited in E. coli and yeast cultures in presence of acetate [21] and ethanol [20] respectively. Moreover, the formation of these by-products constitutes a diversion of carbon that might have contributed to biomass or protein synthesis. Thus, overflow metabolism can pose a major problem in large-scale production of biomass or recombinant proteins [9] [15].

Cultivation of $E$. coli, yeasts, and other microorganisms can be done in a chemostat. The chemostat, introduced in the 1950s independently by Monod [23] and Novick and Szilard [25], is a perfectly mixed reactor, permanently fed with a nutrient rich medium and simultaneously emptied so that the culture volume is kept constant. Using the chemostat is a way to maintain indefinitely a non-zero growth rate, and therefore to study the organisms under various constant growth rates. The classical chemostat model describes the dynamics of a single population with growth limited by a single nutrient. We refer the reader to [28] and [1] for the theory of the chemostat and for different variations of the classical chemostat model. In [33], [32], [16], and [14], the authors study the dynamics of chemostat models with the production of a toxic by-product. In [32], the production of the by-product is described as a consequence of overflow metabolism. However, in all these works the authors assume that the secretion of the by-product occurs at any growth rate while experimental evidence shows that by-product secretion does not take place at low growth rates [4].

In this paper, we study the long-term behavior of a chemostat model accounting for the following features of overflow metabolism:

- secretion of a by-product when the substrate uptake rate is above a threshold;

- biomass loss due to secretion of the by-product;

- inhibition of substrate uptake in presence of the by-product.

The model is mainly inspired by the recently proposed model in [22] that describes the growth of an E. coli culture producing a recombinant protein. In contrast to our model, the authors in [22] consider growth on the by-product (acetate) and an additional variable describing the dynamics of a recombinant protein concentration. E. coli consume acetate only after the glucose (substrate) is totally consumed, phenomenon known as carbon catabolite repression [31]. Thus, given the continuous supply of substrate in chemostats, we neglect the consumption of the by-product in our model. Note that carbon catabolite repression is also observed in yeasts [11]. With respect to the recombinant protein 


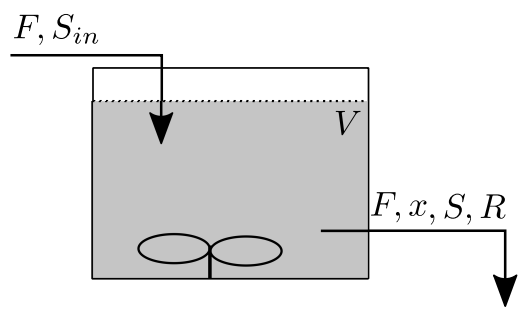

Figure 1: A schematic diagram of a chemostat reactor. The chemostat is fed at a rate $F$ with a substrate concentration $S_{i n}$. The reactor is emptied at the rate $F$ keeping a constant volume $V$. The concentrations of microorganisms $(x)$, substrate $(S)$, and by-product $(R)$ are homogeneous in the medium.

concentration, in the discussion section we show that our results can be easily extended when considering the dynamics of a recombinant protein.

The chemostat model with overflow metabolism is described by an autonomous system of ordinary differential equations. Using a conservation principle, the model can be reduced to a planar system. Thus, we study the dynamics of the planar system by finding appropriate invariant sets and using results on cooperative systems [27]. To extend our results to the original model, we use the well known Theorem of Butler-McGehee [28]. This technique requires the stability of equilibria, which may be difficult to obtain due to the non-smoothness of the by-product excretion rate function (overflow metabolism). This situation is treated with classical results of the theory of differential equations such as the comparison method [7].

This paper is organized as follows. In Section 2, we describe the chemostat model and the main hypotheses. Sections 3 and 4 are devoted to the mathematical analysis of the model. In Section 3, we characterize the existence of equilibria and their local stability. In Section 4, we present the results on the global behavior of the model. The main result is given in this section (Teorem 4.6). In the last section, Section 5, we begin presenting a brief summary of our mathematical results. Then, we finish the paper with a discussion on the steady state production of biomass and recombinant proteins in chemostat-type systems.

\section{Chemostat model}

We consider a chemostat (see Figure 1) with a single population of microorganisms whose concentration is denoted by $x$. This population grows at a specific growth rate $\mu(\cdot)$. The specific growth rate considers the carbon gain by substrate uptake and the carbon loss due to metabolic overflow i.e.

$$
\mu(\cdot)=Y_{S} r_{S}(\cdot)-Y_{R} r_{o f}(\cdot),
$$




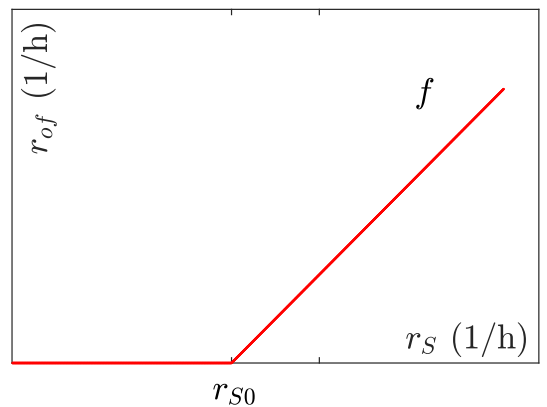

Figure 2: By-product excretion rate $\left(r_{o f}\right)$ as a function of the substrate uptake rate $\left(r_{S}\right)$.

where $r_{S}$ is the substrate uptake rate, $r_{o f}$ is the metabolic overflow rate (or byproduct formation rate), and $Y_{S}, Y_{R}$ are yield coefficients. Following [4], when $r_{S}$ is higher than a threshold rate $r_{S 0}$, then the excretion of by-product occurs at a rate proportional to the difference between $r_{S}$ and $r_{S 0}$ i.e. $r_{o f}=f\left(r_{S}\right)$ with $f$ defined as (see Figure 2):

$$
f\left(r_{S}\right)=\left\{\begin{array}{lll}
k\left(r_{S}-r_{S 0}\right) & \text { if } & r_{S}>r_{S 0}, \\
0 & \text { if } & r_{S} \leq r_{S 0},
\end{array}\right.
$$

with $k>0$. The substrate uptake rate $r_{S}$ is a function of the substrate bulk concentration $(S)$ and the overflow metabolism by-product $(R)$ i.e. $r_{S}=r_{S}(S, R)$. We assume that $r_{S}$ is continuously differentiable for all $S, R \geq 0$ and that:

$$
r_{S}(0, R)=0, \quad \frac{\partial r_{S}}{\partial S}>0, \quad \text { and } \quad \frac{\partial r_{S}}{\partial R}<0 .
$$

Thus, the by-product $R$ has an inhibitory effect on the substrate uptake rate. An example for $r_{S}$ is given by (see [22]):

$$
r_{S}(S, R)=r_{S, \max } \frac{S}{K_{S}+S} \frac{K_{i, R}}{K_{i, R}+R},
$$

where $r_{S, \max }$ is the maximal substrate uptake rate, $K_{S}$ is a half saturation constant, and $K_{i, R}$ is an inhibition constant. The chemostat is fed at a rate $F>0$ with a substrate concentration $S_{i n}$. The dilution rate is defined as $D=F / V$, with $V$ the volume of the culture. Mass balance equations lead to:

$$
\begin{aligned}
& \frac{d x}{d t}=(\mu(S, R)-D) x, \\
& \frac{d S}{d t}=D\left(S_{i n}-S\right)-r_{S}(S, R) x, \\
& \frac{d R}{d t}=-D R+r_{o f}(S, R) x .
\end{aligned}
$$


Model (3) is that of a standard chemostat with a single species with growth limited by a single substrate, with the added feature that a by-product is produced as a consequence of overflow metabolism.

Throughout the paper we assume:

$$
Y_{S}-k Y_{R}>0 .
$$

This assumption implies that the growth rate function $\mu$ is strictly increasing in $S$ and strictly decreasing in $R$. This follows directly from noting that:

$$
\mu(\cdot)=\left\{\begin{array}{lll}
\left(Y_{S}-k Y_{R}\right) r_{S}(\cdot)+k Y_{R} r_{S 0} & \text { if } \quad r_{S}(\cdot)>r_{S 0}, \\
Y_{S} r_{S}(\cdot) & \text { if } \quad r_{S}(\cdot) \leq r_{S 0} .
\end{array}\right.
$$

Assumption (4) is satisfied by the parameters given in [22]. We also assume that

$$
\left.r_{S}\left(S_{i n}, 0\right)>r_{S 0} \quad \text { (i.e. } r_{o f}\left(S_{i n}, 0\right)>0\right) .
$$

In the long-term operation with presence of microorganisms, the substrate concentration in the medium cannot be higher than $S_{i n}$. Then, if $r_{S}\left(S_{i n}, 0\right) \leq r_{S 0}$, overflow metabolism is not possible in the long-term, and the study of the dynamics of (3) is reduced to that of a classical chemostat model.

Recalling the definition of $\mu$ and combining (4) and (5), we have the following inequality:

$$
\mu\left(S_{i n}, 0\right)>Y_{S} r_{S 0} .
$$

This inequality allows us to consider dilution rates between $Y_{S} r_{S 0}$ and $\mu\left(S_{i n}, 0\right)$. As we will show in the next sections, in the long-term operation, only when $Y_{S} r_{S 0}<D<\mu\left(S_{i n}, 0\right)$ there is presence of the by-product in the culture.

As expected, the domain of biological interest, that is $\mathbb{R}_{+}^{3}:=\{(x, S, R) \in$ $\left.\mathbb{R}^{3} x, S, R \geq 0\right\}$, is positively invariant.

The conservation principle for chemostats is satisfied by the variable $W=$ $x+Y_{S} S+Y_{R} R$ i.e.

$$
\frac{d W}{d t}=D\left(Y_{S} S_{i n}-W\right) .
$$

We can rapidly verify that

$$
W(t)=W(0) e^{-D t}+Y_{S} S_{i n}\left(1-e^{-D t}\right) .
$$

In view of the definition of $W$ we have

$$
0 \leq x(t), Y_{S} S(t), Y_{R} R(t) \leq W(t) .
$$

Since $W(t) \rightarrow Y_{S} S_{\text {in }}$ as $t \rightarrow \infty$, we conclude that (3) is dissipative i.e. solutions of (3) are attracted by the bounded set $\left[0, Y_{S} S_{i n}\right] \times\left[0, S_{i n}\right] \times\left[0, Y_{S} S_{i n} / Y_{R}\right]$. 


\section{$3 \quad$ Existence of steady states and local stability}

Equation (3) admits at most two equilibria. A trivial equilibrium corresponds to the absence of microorganisms. It is given by

$$
E_{0}=\left(0, S_{i n}, 0\right)
$$

and it always exists. The other possible equilibrium is characterized by the presence of microorganisms. The presence of the by-product depends on the dilution rate. The following proposition formally characterizes the existence of this equilibrium.

Proposition 3.1 (Existence of the non-trivial equilibrium).

(a) If $\mu\left(S_{i n}, 0\right)>D$, then (3) admits a unique equilibrium $E^{*}=\left(x^{*}, S^{*}, R^{*}\right)$ with presence of microorganisms (i.e. $x^{*}>0$ ). Moreover,

- If $Y_{S} r_{S 0}>D$, then $R^{*}=0$ and $r_{S}\left(S^{*}, 0\right)<r_{S 0}$.

- If $Y_{S} r_{S 0}=D$, then $R^{*}=0$ and $r_{S}\left(S^{*}, 0\right)=r_{S 0}$.

- If $Y_{S} r_{S 0}<D$, then $R^{*}>0$ and $r_{S}\left(S^{*}, R^{*}\right)>r_{S 0}$.

(b) If $\mu\left(S_{i n}, 0\right) \leq D$, then (3) has no equilibrium with presence of microorganisms.

Proof. Assume that $D \leq Y_{S} r_{S 0}$. In this case, any positive steady state of (3) has no by-product. Indeed, by contradiction, if $\left(x^{*}, S^{*}, R^{*}\right)$ is a positive steady state of (3) with $R^{*}>0$, then $r_{o f}\left(S^{*}, R^{*}\right)=D R^{*} / x^{*}>0$ (from the third equation in (3)). Thus, from the first equation in (3) we obtain:

$$
\mu\left(S^{*}, R^{*}\right)-D=\left(Y_{S}-k Y_{R}\right) \underbrace{\left(r_{S}\left(S^{*}, R^{*}\right)-r_{S 0}\right)}_{>0}+\underbrace{Y_{S} r_{S 0}-D}_{\geq 0}>0,
$$

which contradicts the fact that $\left(x^{*}, S^{*}, R^{*}\right)$ is a positive steady state. Hence, any positive steady state of (3) has the form $\left(x^{*}, S^{*}, 0\right)$. As in a classical chemostat model (note that $S \longmapsto \mu(S, 0)$ is strictly increasing), (3) admits a unique positive steady state if $\mu\left(S_{i n}, 0\right)>D$, and has no positive steady states if $\mu\left(S_{i n}, 0\right) \leq D$.

Now assume that $D>Y_{S} r_{S 0}$. In this case, the by-product is present in any positive equilibrium of (3). Indeed, by contradiction, if $\left(x^{*}, S^{*}, 0\right)$ is a positive steady state of $(3)$, then $r_{o f}\left(S^{*}, 0\right)=0$. But we have $Y_{S} r_{S}\left(S^{*}, 0\right)=$ $D>Y_{S} r_{S 0}$, which implies $r_{S}\left(S^{*}, 0\right)>r_{S 0}$, and hence $r_{o f}\left(S^{*}, 0\right)>0$, which is a contradiction. Then, we look for positive steady states $\left(x^{*}, S^{*}, R^{*}\right)$ with $R^{*}>0$. If $R^{*}>0$, then $r_{o f}\left(S^{*}, R^{*}\right)>0$. Thus, we study the following system of equations:

$$
\begin{aligned}
0 & =\left(Y_{S}-k Y_{R}\right) r_{S}(S, R)+k Y_{R} r_{S 0}-D, \\
0 & =D\left(S_{i n}-S\right)-r_{S}(S, R) x, \\
0 & =-D R+k\left[r_{S}(S, R)-r_{S 0}\right] x .
\end{aligned}
$$


From the two first equations in (9), we obtain that:

$$
S=S_{i n}-\beta_{S} x, \beta_{S}:=\frac{1}{D} \frac{D-k Y_{R} r_{S 0}}{Y_{S}-k Y_{R}}>0 .
$$

Combining the three equations in (9), we obtain that $x+Y_{S} S+Y_{R} R=Y_{S} S_{i n}$ (conservation principle, see (6)). Combining this equation with (10), we obtain:

$$
R=\beta_{R} x, \beta_{R}:=\frac{k}{D} \frac{D-Y_{S} r_{S 0}}{Y_{S}-k Y_{R}}>0 .
$$

Combining (10) and (11) with the first equation in (9), we obtain the following equation for $x$ :

$$
\underbrace{\left(Y_{S}-k Y_{R}\right) r_{S}\left(S_{i n}-\beta_{S} x, \beta_{R} x\right)+k Y_{R} r_{S 0}-D}_{f(x)}=0 .
$$

Since $f$ is strictly decreasing, $f(0)=\mu\left(S_{i n}, 0\right)-D$ and $f\left(S_{i n} / \beta_{S}\right)=k Y_{R} r_{S 0}-$ $D<Y_{S} r_{S 0}-D<0$, we conclude that (12) admits a unique solution $x^{*} \in$ $\left(0, S_{i n} / \beta_{S}\right)$ if $\mu\left(S_{i n}, 0\right)>D$, and has no positive solution if $\mu\left(S_{i n}, 0\right) \leq D$. Taking $S^{*}=S_{i n}-\beta_{S} x^{*}$ and $R^{*}=\beta_{R} x^{*}$ we have the positive steady state.

It remains to prove the inequalities in (a). Assume that $\mu\left(S_{i n}, 0\right)>D$ and let $\left(x^{*}, S^{*}, R^{*}\right)$ be the unique positive steady state of (3). If $D \leq Y_{S} r_{S 0}$, then $R^{*}=0$, hence $r_{o f}\left(S^{*}, 0\right)=0$ (from the third equation in (3)). This implies that $Y_{S} r_{S}\left(S^{*}, 0\right)=D$. Thus, if $D<Y_{S} r_{S 0}\left(\right.$ resp. $\left.D=Y_{S} r_{S 0}\right)$, then $r_{S}\left(S^{*}, 0\right)<r_{S 0}\left(\right.$ resp. $\left.r_{S}\left(S^{*}, 0\right)=r_{S 0}\right)$. Now, if $D>Y_{S} r_{S 0}$, then $R^{*}>0$, hence $r_{o f}\left(S^{*}, R^{*}\right)>0$. This implies that $r_{S}\left(S^{*}, R^{*}\right)>r_{S 0}$ and the proof is completed.

The following result shows that the equilibrium with presence of microorganisms is locally stable when $D \neq Y_{S} r_{S 0}$.

Proposition 3.2 (Local stability of $\left.E^{*}\right)$. Assume that $D<\mu\left(S_{i n}, 0\right)$ and let $E^{*}$ be the non-trivial equilibrium given by Proposition 3.1. If $D \neq Y_{S} r_{S 0}$, then $E^{*}$ is locally stable.

Proof. If $D>Y_{S} r_{S 0}$, according to Proposition 3.1, $R^{*}>0$ and $r_{o f}\left(S^{*}, R^{*}\right)>0$. Thus, we can study the local stability of $E^{*}$ in the following system:

$$
\begin{aligned}
\frac{d x}{d t} & =\left[\left(Y_{S}-k Y_{R}\right) r_{S}(S, R)+k Y_{R} r_{S 0}-D\right] x, \\
\frac{d S}{d t} & =D\left(S_{i n}-S\right)-r_{S}(S, R) x \\
\frac{d R}{d t} & =-D R+k\left[r_{S}(S, R)-r_{S 0}\right] x .
\end{aligned}
$$


Set $\alpha:=Y_{S}-k Y_{R}$. The change of variables $U=x+\alpha S$ and $W=x+Y_{S} S+Y_{R} R$ leads (13) to:

$$
\begin{aligned}
\frac{d x}{d t} & =\left[\alpha \hat{r}_{S}(x, U, W)+k Y_{R} r_{S 0}-D\right] x \\
\frac{d U}{d t} & =D\left(\alpha S_{i n}-U\right)+k Y_{R} r_{S 0} x \\
\frac{d W}{d t} & =D\left(Y_{S} S_{i n}-W\right)
\end{aligned}
$$

with

$$
\hat{r}_{S}(x, U, W)=r_{S}\left(\frac{1}{\alpha}(U-x), \frac{1}{Y_{R}}\left(W-\frac{Y_{S}}{\alpha} U\right)+\frac{k}{\alpha} x\right) .
$$

The Jacobian matrix associated with (14) and evaluated at $E^{*}$ is:

$$
J_{1}:=\left[\begin{array}{ccc}
\alpha x^{*} \frac{\partial \hat{r}_{S}}{\partial x} & \alpha x^{*} \frac{\partial \hat{r}_{S}}{\partial U} & \alpha x^{*} \frac{\partial \hat{r}_{S}}{\partial W} \\
k Y_{R} r_{S 0} & -D & 0 \\
0 & 0 & -D
\end{array}\right]
$$

It is clear that one eigenvalue of $J$ is $-D$. The other two eigenvalues are those of the matrix:

$$
J_{1}^{\prime}:=\left[\begin{array}{cc}
\alpha x^{*} \frac{\partial \hat{r}_{S}}{\partial x} & \alpha x^{*} \frac{\partial \hat{r}_{S}}{\partial U} \\
k Y_{R} r_{S 0} & -D
\end{array}\right]
$$

We note that:

$$
\begin{aligned}
& \frac{\partial \hat{r}_{S}}{\partial x}=-\frac{1}{\alpha} \frac{\partial r_{S}}{\partial S}+\frac{k}{\alpha} \frac{\partial r_{S}}{\partial R}<0 \\
& \frac{\partial \hat{r}_{S}}{\partial U}=\frac{1}{\alpha} \frac{\partial r_{S}}{\partial S}-\frac{Y_{S}}{\alpha Y_{R}} \frac{\partial r_{S}}{\partial R}>0 .
\end{aligned}
$$

Thus, it is easy to verify that $\operatorname{Tr}\left(J_{1}^{\prime}\right)<0$ and that $\operatorname{det}\left(J_{1}^{\prime}\right)>\alpha r_{S 0} x \frac{\partial r_{S}}{\partial S}>0$. This implies that both eigenvalues of $J_{1}^{\prime}$ have negative real part. Thus, $E^{*}$ is locally stable.

If $0<D<Y_{S} r_{S 0}$, according to Proposition $3.1, R^{*}=0$ and $r_{o f}\left(S^{*}, 0\right)=0$. Thus, we can study the local stability of $E^{*}$ in the following system:

$$
\begin{aligned}
\frac{d x}{d t} & =\left[Y_{S} r_{S}(S, R)-D\right] x, \\
\frac{d S}{d t} & =D\left(S_{i n}-S\right)-r_{S}(S, R) x, \\
\frac{d R}{d t} & =-D R .
\end{aligned}
$$


The Jacobian matrix associated with $(16)$ and evaluated at $E^{*}$ is:

$$
J_{2}:=\left[\begin{array}{ccc}
0 & x^{*} Y_{S} \frac{\partial r_{S}}{\partial S} & x^{*} Y_{S} \frac{\partial r_{S}}{\partial R} \\
-r_{S} & -D-x^{*} \frac{\partial r_{S}}{\partial S} & -x^{*} \frac{\partial r_{S}}{\partial R} \\
0 & 0 & -D
\end{array}\right] .
$$

As in the previous case, one eigenvalue of $J_{2}$ is $-D$. The other two eigenvalues are those of the matrix:

$$
J_{2}^{\prime}:=\left[\begin{array}{cc}
0 & x^{*} Y_{S} \frac{\partial r_{S}}{\partial S^{\prime}} \\
-r_{S} & -\left(D+x^{*} \frac{\partial r_{S}}{\partial S}\right)
\end{array}\right]
$$

It is clear that $\operatorname{Tr}\left(J_{2}^{\prime}\right)<0$ and $\operatorname{det}\left(J_{2}^{\prime}\right)>0$. Hence, both eigenvalues of $J_{2}^{\prime}$ have negative real part. Thus, $E^{*}$ is locally stable.

\section{Global behavior and main result}

In this section, we aim to prove that if (3) admits an equilibrium with presence of microorganisms, which is unique according to Proposition 3.1, then any solution to (3) approaches it asymptotically, provided a positive initial population. The first result in this section shows the existence of two positively invariant sets, which will be repeatedly used in this section.

Lemma 4.1 (Positively invariant sets).

(a) The set $\Omega_{1}:=\left\{(x, S, R) \in \mathbb{R}_{+}^{3} ; x+Y_{S} S \leq Y_{S} S_{i n}\right\}$ is positively invariant.

(b) If $D \geq Y_{S} r_{S 0}$, the set $\Omega_{2}:=\Omega_{1} \cap\left\{(x, S, R) ; r_{S}(S, R) \geq r_{S 0}\right\}$ is positively invariant.

Proof. We have that the variable $V:=x+Y_{S} S$ satisfies the following differential equation:

$$
\frac{d V}{d t}=D\left(Y_{S} S_{i n}-V\right)-Y_{R} r_{o f}(S, R) x
$$

The proof of (a) follows from the fact that $\left.\frac{d V}{d t}\right|_{V=Y_{S} S_{i n}} \leq 0$ and $\mathbb{R}_{+}^{3}$ is positively invariant. For (b), let us consider the variable $y=r_{S}(S, R)$. Then we have:

$$
\frac{d y}{d t}=\left(D\left(S_{i n}-S\right)-y x\right) \frac{\partial r_{S}(S, R)}{\partial S}+\left(-D R+r_{o f}(S, R) x\right) \frac{\partial r_{S}(S, R)}{\partial R}
$$

Since $\Omega_{1}$ is positively invariant, it is enough to show that $\left.\frac{d y}{d t}\right|_{y=r_{S 0}} \geq 0$ whenever $(x, S, R) \in \Omega_{1}$. Indeed, we have

$$
\left.\frac{d y}{d t}\right|_{y=r_{S 0}}=\left(D\left(S_{i n}-S\right)-x Y_{S} r_{S 0}\right) \frac{\partial r_{S}(S, R)}{\partial S}-D R \frac{\partial r_{S}(S, R)}{\partial R} .
$$


Since $\frac{\partial r_{S}(S, R)}{\partial S}>0, \frac{\partial r_{S}(S, R)}{\partial R}<0, Y_{S} S+x \leq Y_{S} S_{i n}$ (inside $\Omega_{1}$ ), and $D \geq Y_{S} r_{S 0}$, we obtain

$$
\begin{aligned}
\left.\frac{d y}{d t}\right|_{y=r_{S 0}} & >\left(Y_{S} r_{S 0}\left(S_{i n}-S\right)-r_{S 0} x\right) \frac{\partial r_{S}(S, R)}{\partial S} \\
& =r_{S 0}\left(Y_{S} S_{i n}-x-Y_{S} S\right) \frac{\partial r_{S}(S, R)}{\partial S} \\
& \geq 0
\end{aligned}
$$

This completes the proof.

The following result shows that if there is no equilibrium with presence of microorganisms (i.e. $\left.\mu\left(S_{i n}, 0\right) \leq D\right)$, then the population goes to extinction.

Proposition 4.2 (Extinction). Let $E_{0}$ be given by (8). If $\mu\left(S_{i n}, 0\right) \leq D$, then any solution of (3) approaches $E_{0}$ asymptotically.

Proof. Let $(\tilde{x}, \tilde{S}, \tilde{R})$ be a solution of $(3)$, and let $\tilde{V}=\tilde{x}+Y_{S} \tilde{S}$. We have that

$$
\frac{d \tilde{V}}{d t}=D\left(Y_{S} S_{i n}-\tilde{V}\right)-r_{o f}(\tilde{S}, \tilde{R}) \tilde{x} .
$$

We can easily verify that :

$$
\begin{aligned}
& \frac{d \tilde{x}}{d t} \leq f_{1}(\tilde{x}, \tilde{V}):=\left[\mu\left(\frac{\tilde{V}-\tilde{x}}{Y_{S}}, 0\right)-D\right] \tilde{x}, \\
& \frac{d \tilde{V}}{d t} \leq f_{2}(\tilde{x}, \tilde{V}):=D\left(Y_{S} S_{i n}-\tilde{V}\right) .
\end{aligned}
$$

Now, let $(\hat{x}, \hat{V})$ be the unique solution of

$$
\begin{aligned}
& \frac{d x}{d t}=f_{1}(x, V), \\
& \frac{d V}{d t}=f_{2}(x, V),
\end{aligned}
$$

satisfying $\tilde{x}(0)=\hat{x}(0)$ and $\tilde{V}(0)=\hat{V}(0)$. We note that (19) is cooperative i.e. $x \longmapsto f_{2}(x, V)$ and $V \longmapsto f_{1}(x, V)$ are increasing [28]. Then, applying Theorem B.1 from Appendix B in [28], we conclude that

$$
\tilde{x}(t) \leq \hat{x}(t) \text { and } \tilde{V}(t) \leq \hat{V}(t) \text { for all } t \geq 0 .
$$

Again, due to the cooperativity of (19) we conclude that $(\hat{x}, \hat{V})$ approaches an equilibrium asymptotically. Since the unique equilibrium of $(19)$ is $\left(0, Y_{S} S_{i n}\right)$, we conclude that $(\hat{x}, \hat{V})$ approaches $\left(0, Y_{S} S_{i n}\right)$ asymptotically. From $(20), \tilde{x}$ approaches 0 asymptotically. Now, noting that $\tilde{S}(t) \leq \tilde{V}(t) / Y_{S}$ for all $t \geq 0$, we have that

$$
\frac{d \tilde{V}}{d t} \geq g(t, \tilde{V}):=D\left(Y_{S} S_{i n}-\tilde{V}\right)-Y_{R} r_{o f}\left(\tilde{V} / Y_{S}, 0\right) \tilde{x}(t)
$$


Let $\underline{V}$ be the unique solution of:

$$
\frac{d V}{d t}=g(t, V)
$$

satisfying $\underline{V}(0)=\tilde{V}(0)$. Thus, by a comparison theorem argument, we conclude that $\underline{V}(t) \leq \tilde{V}(t)$ for all $t \geq 0$. Let us define $g_{0}(V):=D\left(Y_{S} S_{\text {in }}-V\right)$. Since $\left|g(t, V)-g_{0}(V)\right|=Y_{R} r_{o f}(V, 0) \tilde{x}(t) \rightarrow 0$ as $t \rightarrow \infty$, we can apply Theorem 1.2 in [29] and conclude that $\underline{V}$ approaches $Y_{S} S_{i n}$ asymptotically. Now, since $\underline{V}(t) \leq$ $\tilde{V}(t) \leq \hat{V}(t)$ for all $t \geq 0$, we conclude that $\tilde{V}$ approaches $Y_{S} S_{i n}$ asymptotically. Finally, consider the variable $\tilde{W}=\tilde{x}+Y_{S} \tilde{S}+Y_{R} \tilde{R}$. In view of (6), $\tilde{W}$ converges to $Y_{S} S_{i n}$. Consequently, $\tilde{R}=\frac{\tilde{W}-\tilde{V}}{Y_{R}}$ converges to 0 , and the proof is complete.

In view of (6), the solutions of (3) approach the hyperplane:

$$
\Omega:=\left\{(x, S, R) \in \mathbb{R}_{+}^{3} ; x+Y_{S} S+Y_{R} R=Y_{S} S_{i n}\right\}
$$

The set $\Omega$ is positively invariant with respect to (3). This implies that the dynamics of solutions starting in $\Omega$ correspond to that of a two-dimensional system. The following two results describe the dynamics of any solution starting in $\Omega$.

Lemma 4.3. If $\mu\left(S_{i n}, 0\right)>D$ and $D>Y_{S} r_{S 0}$, for any solution $(x, S, R)$ to (3) starting on $\Omega$ with $x(0)>0$, there exists $t^{\prime}$ such that $r_{\text {of }}(S(t), R(t)) \geq 0$ for all $t \geq t^{\prime}$.

Proof. Let $(\tilde{x}, \tilde{S}, \tilde{R})$ be a solution of $(3)$ with $\tilde{x}(0)>0$ and $(\tilde{x}(0), \tilde{S}(0), \tilde{R}(0)) \in$ $\Omega$, and let $\Omega_{1}$ and $\Omega_{2}$ be the positively invariant sets defined in Lemma 4.1. Since $(\tilde{x}, \tilde{S}, \tilde{R})$ starts in $\Omega$, we have that $\tilde{x}(t)+Y_{S} \tilde{S}(t)=Y_{S} S_{i n}-Y_{R} \tilde{R}(t) \leq Y_{S} S_{\text {in }}$ for all $t \geq 0$. Hence, $(\tilde{x}(t), \tilde{S}(t), \tilde{R}(t)) \in \Omega_{1}$ for all $t \geq 0$. Now, we claim the existence of $t^{\prime}>0$ such that $r_{S}\left(\tilde{S}\left(t^{\prime}\right), \tilde{R}\left(t^{\prime}\right)\right) \geq r_{S 0}$. Indeed, by contradiction, let us assume that $r_{S}(\tilde{S}(t), \tilde{R}(t))<r_{S 0}$ for all $t \geq 0$. Consider the variable $\tilde{V}=\tilde{x}+Y_{S} \tilde{S}$. Then $(\tilde{x}, \tilde{V})$ is a solution of the following system:

$$
\begin{aligned}
\frac{d x}{d t} & =\left(Y_{S} r_{S}\left(\frac{V-x}{Y_{S}}, \frac{Y_{S} S_{i n}-V}{Y_{R}}\right)-D\right) x, \\
\frac{d V}{d t} & =D\left(Y_{S} S_{i n}-V\right) .
\end{aligned}
$$

The planar system (24) is cooperative, then $(\tilde{x}, \tilde{V})$ approaches a steady state asymptotically. Let $E^{*}=\left(x^{*}, S^{*}, R^{*}\right)$ be given by Proposition 3.1. System (24) admits two equilibria, $F_{0}=\left(0, Y_{S} S_{i n}\right)$ and $F^{*}=\left(x^{*}, Y_{S} S_{i n}\right)$. Thus, $r_{S}(\tilde{S}(t), \tilde{R}(t))$ approaches either $r_{S}\left(S_{i n}, 0\right)\left(>r_{S 0}\right)$ or $D / Y_{S}\left(>r_{S 0}\right)$. This implies that $(\tilde{x}, \tilde{S}, \tilde{R})$ enters $\Omega_{2}$ which contradicts our hypothesis. Then, there is $t^{\prime}>0$ such that $r_{S}\left(\tilde{S}\left(t^{\prime}\right), \tilde{R}\left(t^{\prime}\right)\right)>r_{S 0}$ i.e. $\left(\tilde{x}\left(t^{\prime}\right), \tilde{S}\left(t^{\prime}\right), \tilde{R}\left(t^{\prime}\right)\right) \in \Omega_{2}$. Since $\Omega_{2}$ is positively invariant, the proof is complete. 
The following result describes the global behavior of solutions of (3) starting on $\Omega$.

Proposition 4.4. Assume that $\mu\left(S_{i n}, 0\right)>D$, and let $E^{*}$ and $E_{0}$ be the equilibria given by Proposition 3.1 and (8) respectively. Then, for any solution $(x, S, R)$ to (3) starting on $\Omega$ we have

(a) if $x(0)>0$, then $(x(t), S(t), R(t)) \rightarrow E^{*}$ as $t \rightarrow \infty$,

(b) if $x(0)=0$, then $(x(t), S(t), R(t)) \rightarrow E_{0}$ as $t \rightarrow \infty$.

Proof. Let $(\tilde{x}, \tilde{S}, \tilde{R})$ be a solution of $(3)$ with $(\tilde{x}(0), \tilde{S}(0), \tilde{R}(0)) \in \Omega$. To prove (a), let us assume that $\tilde{x}(0)>0$. It is not difficult to see that $(\tilde{x}, \tilde{R})$ is a solution of the following system:

$$
\begin{aligned}
& \frac{d x}{d t}=\left(\mu\left(\varphi_{S}(x, R), R\right)-D\right) x \\
& \frac{d R}{d t}=-D R+r_{o f}\left(\varphi_{S}(x, R), R\right) x
\end{aligned}
$$

with $\varphi_{S}(x, R)=S_{i n}-\left(Y_{R} R+x\right) / Y_{S}$. If $D \leq Y_{S} r_{S 0}$, then (25) admits only two equilbria, $F_{0}=(0,0)$ and $F^{*}=\left(x^{*}, 0\right)$. Hence, $(25)$ has no interior steady states, and consequently (in a planar system) no limit cycles. The Jacobian matrix of (25) evaluated at $F_{0}$ is:

$$
\left[\begin{array}{cc}
\mu\left(S_{i n}, 0\right)-D & 0 \\
0 & -D
\end{array}\right] .
$$

Since $\mu\left(S_{i n}, 0\right)-D>0, F_{0}$ is a saddle point which can only be reached if $\tilde{x}(0)=0$. Consequently, by the Poincaré - Bendixson theorem (Theorem 2 in Chapter 3.7 in $[26]),(\tilde{x}, \tilde{R})$ approaches $F^{*}$ asymptotically, and hence $(\tilde{x}, \tilde{S}, \tilde{R})$ approaches $E^{*}$ asymptotically. Now, let us assume that $D>Y_{S} r_{S 0}$. From Lemma 4.3, we can assume that $r_{S}(\tilde{S}(t), \tilde{R}(t)) \geq r_{S 0}$ for all $t \geq 0$. Thus, $(\tilde{x}, \tilde{S}, \tilde{R})$ is a solution of:

$$
\begin{aligned}
\frac{d x}{d t} & =\left(\left(Y_{S}-k Y_{R}\right) r_{S}(S, R)+k Y_{R} r_{S 0}-D\right) x \\
\frac{d S}{d t} & =D\left(S_{i n}-S\right)-r_{S}(S, R) x \\
0 & =Y_{S}\left(S_{i n}-S\right)-Y_{R} R-x .
\end{aligned}
$$

Set $\alpha:=Y_{S}-k Y_{R}$ and consider the variable $\tilde{U}=\tilde{x}+\alpha \tilde{S}$. Then $(\tilde{x}, \tilde{U})$ is a solution of the following system:

$$
\begin{aligned}
\frac{d x}{d t} & =\left(\alpha r_{S}\left(\varphi_{S}(x, U), \varphi_{R}(x, U)\right)+k Y_{R} r_{S 0}-D\right) x \\
\frac{d U}{d t} & =D\left(\alpha S_{i n}-U\right)+k Y_{R} r_{S 0} x
\end{aligned}
$$


with

$$
\varphi_{S}(x, U)=\frac{U-x}{\alpha} \text { and } \varphi_{R}(x, U)=\frac{1}{Y_{R}}\left(Y_{S} S_{i n}-\frac{Y_{S} U}{\alpha}\right)+\frac{k x}{\alpha} .
$$

Since $(27)$ is a planar cooperative system, $(\tilde{x}(t), \tilde{U}(t))$ approaches either $F^{*}=$ $\left(x^{*}, x^{*}+\alpha S^{*}\right)$ or $F_{0}=\left(0, \alpha S_{i n}\right)$. The Jacobian matrix of (27) evaluated at $F_{0}$ is:

$$
\left[\begin{array}{cc}
\mu\left(S_{i n}, 0\right)-D & 0 \\
k Y_{R} r_{S 0} & -D
\end{array}\right]
$$

It is clear that $F_{0}$ is a saddle point which can only be reached if $\tilde{x}(0)=0$. Consequently, as in the previous case, $(\tilde{x}, \tilde{S}, \tilde{R})$ approaches $E^{*}$ asymptotically. This completes the proof of part (a). For (b), let us assume that $\tilde{x}(0)=0$. Then, $\tilde{x}(t)=0$ for all $t \geq 0$. This implies that $\frac{d \tilde{S}}{d t}=D\left(\tilde{S}-S_{i n}\right)$ and $\frac{d \tilde{R}}{d t}=-D \tilde{R}$. Consequently $(\tilde{x}, \tilde{S}, \tilde{R})$ approaches $E_{0}$ asymptotically.

According to Proposition 3.1, if $D=Y_{S} r_{S 0}$, then the positive equilibrium $E^{*}=\left(x^{*}, S^{*}, 0\right)$ satisfies $r_{S}\left(S^{*}, 0\right)=r_{S 0}$ and the function $r_{o f}$ is not differentiable at $\left(S^{*}, 0\right)$. This poses a problem for the study of the local stability of $E^{*}$, and consequently for the application of classical arguments (e.g. Butler-McGehe Theorem) to extend Proposition 4.4 to any initial condition. The following result considers this particular case.

Proposition 4.5. Assume that $\mu\left(S_{i n}, 0\right)>D$ and let $E^{*}$ be given by Proposition 3.1. If $D=Y_{S} r_{S 0}$, then $E^{*}$ is stable.

Proof. Let $\xi(t)=(x(t), S(t), R(t))$ be a solution of $(3)$ with $x(0)>0$ and $S(0), R(0) \geq 0$, and let $\Omega_{1}$ and $\Omega_{2}$ be the sets defined in Lemma 4.1. Consider the following sets:

- $\Omega_{1}^{\prime}:=\left\{(x, S, R) \in \mathbb{R}_{+}^{3} ; x+Y_{S} S>Y_{S} S_{i n}\right\}$,

- $\Omega_{2}^{\prime}:=\left\{(x, S, R) \in \mathbb{R}_{+}^{3} ; r_{S}(S, R)<r_{S 0}\right\} \cap \Omega_{1}$.

Given sets $A, B \in\left\{\Omega_{1}^{\prime}, \Omega_{2}^{\prime}, \Omega_{2}\right\}$, we will say that $\xi$ moves from $A$ to $B$, if there are $t^{\prime} \geq 0$ and $\tau>0$ such that $\xi(t) \in A$ for all $t \in\left(t^{\prime}-\tau, t^{\prime}\right), \xi\left(t^{\prime}\right) \in A \cup B$, and $\xi(t) \in B-A$ for all $t \in\left(t^{\prime}, t^{\prime}+\tau\right)$. This means that if $\xi$ moves from $A$ to $B$, then there is a time when $\xi$ is in $A$ and then later is in $B$ but not in $A$. Since $\Omega_{1}$ and $\Omega_{2}$ are positively invariant (see Lemma 4.1), $\xi$ can only move from $\Omega_{1}^{\prime}$ to $\Omega_{2}^{\prime}$, from $\Omega_{1}^{\prime}$ to $\Omega_{2}$, or from $\Omega_{2}^{\prime}$ to $\Omega_{2}$. Hence, $\xi$ has one of the following global behaviors:

a) $\xi(t) \in A$ for all $t \geq 0$ with $A \in\left\{\Omega_{1}^{\prime}, \Omega_{2}^{\prime}, \Omega_{2}\right\}$,

b) $\xi$ starts on $\Omega_{1}^{\prime}$ and moves either to $\Omega_{2}^{\prime}$ or to $\Omega_{2}$,

c) $\xi$ starts on $\Omega_{2}^{\prime}$ and moves to $\Omega_{2}$,

d) $\xi$ starts on $\Omega_{1}^{\prime}$, then moves to $\Omega_{2}^{\prime}$, and then to $\Omega_{2}$. 
Let $\epsilon>0$ be given. We have to prove the existence of a $\delta>0$ such that in any situation listed above, if $\left\|\xi(0)-E^{*}\right\|<\delta$ then $\left\|\xi(t)-E^{*}\right\|<\epsilon$ for all $t \geq 0$. We only give the proof in the situation $\mathrm{d}$ ) because the proof in the other situations is almost the same. Thus, let us assume the existence of $t_{1}, t_{2}>0$ such that $t_{1}<t_{2}$ and $\xi(t) \in \Omega_{1}^{\prime}$ for all $t \in\left[0, t_{1}\right), \xi(t) \in \Omega_{2}^{\prime}$ for all $t \in\left[t_{1}, t_{2}\right)$, and $\xi(t) \in \Omega_{2}$ for all $t \geq t_{2}$. For all $t \geq t_{2}, \xi(t)$ can be seen as a solution of (13). In such a case, we can study the Jacobian matrix of (13) evaluated at $E^{*}$ (as done in the proof of Proposition 3.2) to conclude the existence of $\delta_{2}>0$ such that $\left\|\xi(t)-E^{*}\right\|<\epsilon$ for all $t \geq t_{2}$ provided $\left\|\xi\left(t_{2}\right)-E^{*}\right\|<\delta_{2}$. Now for all $t \in\left[t_{1}, t_{2}\right)$, $\xi(t)$ can be seen as a solution of (16). In such a case, we can study the Jacobian matrix of (16) evaluated at $E^{*}$ to conclude the existence of $\delta_{1}>0$ such that $\left\|\xi(t)-E^{*}\right\|<\delta_{2} / 2$ for all $t \in\left[t_{1}, t_{2}\right)$ provided $\left\|\xi\left(t_{1}\right)-E^{*}\right\|<\delta_{1}$. Finally, for all $t \in\left[0, t_{1}\right)$, consider the variables $V=x+Y_{S} S$ and $W=x+Y_{S} S+Y_{R} R$. It is clear that:

$$
\frac{d V}{d t}=D\left(Y_{S} S_{i n}-V\right)-Y_{R} r_{o f}(S, R) x \leq D\left(Y_{S} S_{i n}-V\right) .
$$

Using the definition of $\Omega_{1}^{\prime}$ and (28) we obtain:

$$
0 \leq V(t)-Y_{S} S_{i n} \leq V(0)-Y_{S} S_{i n} .
$$

Again, using the definition of $\Omega_{1}^{\prime}$ and (7), we obtain that

$$
0 \leq W(t)-Y_{S} S_{i n} \leq W(0)-Y_{S} S_{i n}
$$

Since $S=(V-x) / Y_{S}$ and $R=(W-V) / Y_{R}$, we have that:

$$
x h\left(W(0)-Y_{S} S_{i n}, x\right) \leq \frac{d x}{d t} \leq x g\left(V(0)-Y_{S} S_{i n}, x\right),
$$

with $g(v, x)=\mu\left(S_{i n}+v / Y_{S}-x / Y_{S}, 0\right)-D$ and $h(v, x)=\mu\left(S_{i n}-x / Y_{S}, v / Y_{R}\right)-$ $D$. We note that $g$ is strictly increasing in $v$ and strictly decreasing in $x$ and that $g(0,0)>0$. Moreover, for $M(v):=v+Y_{S} S_{i n}$ we have $g(v, M(v))=-D<0$. Similarly, $h$ is strictly decreasing in both, $v$ and $x$. It is also clear that $h(0,0)>0$ and $h\left(v, Y_{S} S_{i n}\right)=-D<0$. Applying Lemmas 5.1 and 5.2 in the Appendix, for any $\epsilon^{\prime}>0$ there is $v_{\epsilon^{\prime}}$ such that $\left|x(t)-x^{*}\right|<\epsilon^{\prime}$ provided $\left|x(0)-x^{*}\right|<\epsilon^{\prime} / 2$, $V(0)-Y_{S} S_{i n}<v_{\epsilon^{\prime}}$, and $W(0)-Y_{S} S_{i n}<v_{\epsilon^{\prime}}$. Thus, from (29) and (30), we conclude that $\left|x(t)-x^{*}\right|<\epsilon^{\prime},\left|V(t)-Y_{S} S_{i n}\right|<\epsilon^{\prime}$ and $\left|W(t)-Y_{S} S_{i n}\right|<\epsilon^{\prime}$ for all $t \in\left[0, t_{1}\right]$, provided

$$
\left|x(0)-x^{*}\right|<\epsilon /^{\prime} 2 \text {, and }\left|V(0)-Y_{S} S_{i n}\right|,\left|W(0)-Y_{S} S_{i n}\right|<\min \left\{v_{\epsilon^{\prime}}, \epsilon^{\prime}\right\} .
$$

Choosing an appropriate $\epsilon^{\prime}$, and writing $S$ and $R$ in terms of $x, V$, and $W$, we can find $\delta\left(\epsilon^{\prime}\right)$ such that $\left\|\xi(t)-E^{*}\right\|<\delta_{1} / 2$ for all $t \in\left[0, t_{1}\right)$ provided $\left\|\xi(t)-E^{*}\right\|<\delta\left(\epsilon^{\prime}\right)$. Since $\xi$ is continuous, we conclude that $\left\|\xi(t)-E^{*}\right\|<\epsilon$ for all $t \geq 0$ provided $\left\|\xi(t)-E^{*}\right\|<\delta\left(\epsilon^{\prime}\right)$.

Theorem 4.6 (Main result). Let $E^{*}$ and $E_{0}$ be the equilibria given by Proposition 3.1 and (8) respectively. We have: 
a) If $\mu\left(S_{\text {in }}, 0\right)>D$, then $E^{*}$ is globally asymptotically stable on $(0, \infty) \times \mathbb{R}_{+}^{2}$.

b) If $\mu\left(S_{i n}, 0\right) \leq D$, then any solution to (3) approaches $E_{0}$ asymptotically.

Proof. Part (b) follows directly from Proposition 4.2. For (a), let $(x, S, R)$ be a solution of (3) with $x(0)>0, S(0), R(0) \geq 0$. Let us write $P=(x(0), S(0), R(0))$. In view of $(7)$, we have that $\omega(P) \subset \Omega$, where $\omega(P)$ denotes the $\omega$-limit set of $P$ and $\Omega$ is defined in (23). From Proposition 4.4, the $\omega$-limit set of any trajectory passing through $\Omega$ is either $E_{0}$ or $E^{*}$. Consequently,

$$
\omega(P) \cap\left\{E_{0}, E^{*}\right\} \neq \emptyset .
$$

The Jacobian matrix associated with (3) and evaluated at $E_{0}$ is:

$$
J:=\left[\begin{array}{ccc}
\mu\left(S_{i n}, 0\right)-D & 0 & 0 \\
-r_{S}\left(S_{i n}, 0\right) & -D & 0 \\
r_{o f}\left(S_{i n}, 0\right) & 0 & -D
\end{array}\right] .
$$

It is clear that $J$ has two negative eigenvalues and one positive eigenvalue. Let $\Omega_{0}$ be the two-dimensional subspace spanned by the eigenvectors corresponding to the negative eigenvalues i.e. $\Omega_{0}:=\{0\} \times \mathbb{R}_{+}^{2}$. It is clear that $\Omega_{0}$ is positively invariant and that any solution starting on $\Omega_{0}$ approaches $E_{0}$ asymptotically. Since $\Omega_{0}$ is a manifold trivially tangent to $\Omega_{0}$ at 0 , we conclude that $\Omega_{0}$ is the stable (global) manifold of (3) at $E_{0}$ (see Chapter 2.7 in [26]). Since $P \notin \Omega_{0}$, we have that $\omega(P) \neq\left\{E_{0}\right\}$. Now, let us assume that $E_{0} \in \omega(P)$. According to the Theorem of Butler-McGehee (see for example page 12 in $[28]$ ), $\omega(P)$ intersects $\Omega_{0}$ in a point other than $E_{0}$. The (whole) trajectory of that point, say $\left(0, S_{0}, R_{0}\right)$, is given by

$$
\gamma(t)=\left(0, S_{0} e^{-D t}+Y_{S} S_{i n}\left(1-e^{-D t}\right), R_{0} e^{-D t}\right), \quad t \in \mathbb{R} .
$$

It is clear that $\gamma$ is unbounded (as $t \rightarrow-\infty$ ). Consequently, $\omega(P)$ contains an unbounded trajectory. However, $\omega(P)$ is a bounded set because the solutions to (3) are ultimately bounded (see Lemma 3.1.2 in [13]). This contradiction implies that $E_{0}$ cannot be in $\omega(P)$. Hence, from (31), we conclude that $E^{*} \in \omega(P)$. From Propositions 3.2 and 4.5 we have that $E^{*}$ is stable, hence $\omega(P)=\left\{E^{*}\right\}$. This completes the proof.

\section{Discussion}

\subsection{Summary of our mathematical results: Survival, ex- tinction, and stability}

The chemostat with overflow metabolism, described by (3), admits at most two equilibria. An extinction equilibrium, denoted by $E_{0}=\left(0, S_{i n}, 0\right)$, that corresponds to the absence of microorganisms and always exists. The other possible equilibrium, denoted by $E^{*}=\left(x^{*}, S^{*}, R^{*}\right)$, is characterized by the 
Table 1: Kinetic parameters and yield coefficients taken from [22]. $D W$ stands for dry weight.

\begin{tabular}{lll}
\hline Parameter & Value & Unit \\
\hline$r_{S, \max }$ & 1.53 & $h^{-1}$ \\
$K_{S}$ & 0.09 & $g / L$ \\
$K_{i, R}$ & 0.52 & $g / L$ \\
\hline$k$ & 0.17 & - \\
$r_{S 0}$ & 0.7 & $h^{-1}$ \\
\hline$Y_{S}$ & 0.44 & $g D W / g S$ \\
$Y_{R}$ & 0.3 & $g D W / g R$ \\
\hline
\end{tabular}

presence of microorganism i.e. $x^{*}>0$. Our main result (Theorem 4.6), states that if $E^{*}$ exists, then any solution to (3) with a positive initial population approaches (asymptotically) $E^{*}$. That is, given a solution $(x(t), S(t), R(t))$ of (3) with $x(0)>0$, we have that

$$
\lim _{t \rightarrow \infty} x(t)=x^{*}, \quad \lim _{t \rightarrow \infty} S(t)=S^{*}, \quad \text { and } \quad \lim _{t \rightarrow \infty} R(t)=R^{*} .
$$

On the other hand, the non-existence of $E^{*}$ implies that any solution to (3) approaches the extinction equilibrium asymptotically: meaning that $\lim _{t \rightarrow \infty} x(t)=$ 0. Proposition 3.1 in Section 3 gives necessary and sufficient conditions for the existence of $E^{*}$. Indeed, $E^{*}$ exists if and only if $\mu\left(S_{i n}, 0\right)>D$. The survival of microorganisms (existence of $E^{*}$ ) does not ensure the presence of the overflow metabolism by-product in the medium. According to Proposition 3.1, $R^{*}>0$ if and only if $Y_{S} r_{S 0}<D<\mu\left(S_{i n}, 0\right)$.

Overflow metabolism, and the consequent presence of a by-product, does not generate multistability. That is, if $E^{*}$ exists, there are no solutions with positive initial population converging to $E_{0}$. In [32], the authors observed the multiplicity of stable steady states. However, apart from taking $r_{S 0}=0$, they assume that excess of substrate inhibits the growth rate. Thus, the existence of multiple steady states is due to substrate inhibition and not to overflow metabolism.

\subsection{Acetate formation and productivity in $E$. coli cultures}

In E. coli cultures, the by-product corresponds to acetate. According to Proposition 3.1 , the presence of acetate in the non-trivial equilibrium $E^{*}$ depends on the dilution rate. This is illustrated in Figure 3A. Indeed, in presence of bacteria, $R^{*}>0$ if and only if $D>r_{S 0} Y_{S}$. This relation between the acetate steady state concentration and the dilution rate has been observed experimentally in [10]. This may suggest an optimal operation of the chemostat at dilution rates lower than $Y_{S} r_{S 0}$ to avoid the presence of acetate in the culture. Indeed, different authors have shown that preventing acetate formation in fed-batch leads to higher density cultures [19], [2].

To evaluate this strategy in chemostat cultures, let us consider the (steady state) 

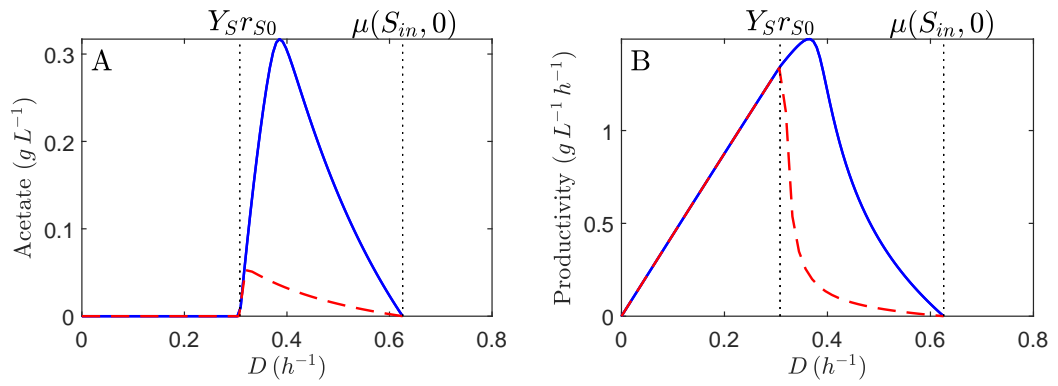

Figure 3: Acetate concentration (A) and productivity (B) evaluated at steady state for different dilution rates. The function $r_{S}$ is taken as in (2). The continuous line is obtained with the parameters from Table 1 . The dashed line is obtained with the parameters from Table 1 after replacing the value of $K_{i, R}$ by $0.052 \mathrm{~g} / L$.

productivity defined as $P^{*}=D x^{*}$, with $x^{*}$ the steady state concentration of bacteria at the dilution rate $D . P^{*}$ quantifies the biomass that is produced per unit of time at steady state. To determine $P^{*}$ numerically, let us assume that $r_{S}$ is given by (2), and consider the parameters estimated by Mauri and collaborators in [22] (see Table 1). Figure 3B shows that the steady state productivity is maximal at a value of the dilution rate higher than $Y_{S} r_{S 0}$ (continuous line). This suggest that preventing acetate formation is not a good strategy in chemostat cultures, in contrast to fed-batch cultures. The veracity of this observation depends on the choice of parameters. For instance, for low values of $K_{i, R}$ (strong inhibition), the maximal productivity is reached at $D=Y_{S} r_{S 0}$ (see dashed line in Figure 3).

As shown in Figure 3B (continuous line), maximal productivity of the system is accompanied by the secretion of acetate. A natural strategy to increase this maximal productivity is removing acetate from the culture during fermentation. This can be done with a dialysis reactor [24], or with macroporous ion-exchange resins [17]. However, these methods tend to remove nutrients that are necessary for cell growth. A promising alternative consists in introducing an additional $E$. coli strain (a cleaner), which has been metabolically engineered to consume acetate. Thus, two different E. coli populations coexist in the culture: one producing biomass, and one reducing the presence of acetate. Experimental results have shown an increase of the productivity with this strategy [5]. A few mathematical works have studied the dynamics of such communities [16] [14]. However, as mentioned in the introduction, the authors assume that overflow metabolism always occur (i.e. $r_{S 0}=0$ ). Thus, our results give a basis to understand the dynamics of such microbial communities when $r_{S 0}>0$. 


\subsection{Recombinant protein production}

Following [22], and using the notation of this paper, the dynamics of a recombinant protein, which concentration is denoted by $H$, follows from:

$$
\begin{aligned}
& \frac{d H}{d t}=Y_{H} \mu(S, R) x-D H, \\
& \frac{d x}{d t}=\left[\left(1-Y_{H}\right) \mu(S, R)-D\right] x, \\
& \frac{d S}{d t}=D\left(S_{i n}-S\right)-r_{S}(S, R) x, \\
& \frac{d R}{d t}=-D R+r_{o f}(S, R) x .
\end{aligned}
$$

Here, $Y_{H}$ is the protein yield coefficient representing the carbon diversion to protein production. Let $(H, x, S, R)$ be a solution of (32) with $x(0)>0$, $H(0), S(0), R(0) \geq 0$. The dynamics of $(x, S, R)$ is independent of $H$ and can be described by Theorem $4.6^{2}$. Indeed, if $\left(1-Y_{H}\right) \mu\left(S_{i n}, 0\right)>D$, then there is $x^{*}>0$ such that $\lim _{t \rightarrow \infty} x(t)=x^{*}$. Now, it is easy to verify that the variable $y:=\frac{Y_{H}}{1-Y_{H}} x-H$ satisfies $\frac{d y}{d t}=-D y$. Therefore, $\lim _{t \rightarrow \infty} y(t)=0$, which implies that $\lim _{t \rightarrow \infty} H(t)=\frac{Y_{H}}{1-Y_{H}} x^{*}$. Thus, we define the steady state protein productivity as:

$$
P_{H}^{*}=D \frac{Y_{H}}{1-Y_{H}} x^{*}
$$

Note that the value of $x^{*}$ depends on the values of $Y_{H}$ and $D$ and that $P_{H}^{*}$ only exists if $0<D<\left(1-Y_{H}\right) \mu\left(S_{i n}, 0\right)$. These results allow to illustrate the impact of $Y_{H}$ on the protein productivity. If $Y_{H}=0$, there is no production of $H$, and consequently $P_{H}^{*}=0$. On the other hand, if $Y_{H}$ approaches 1 , it can be shown that $P_{H}^{*}$ approaches 0 . Indeed, using the restriction over $D$ we obtain $P_{H}^{*}<\mu\left(S_{i n}, 0\right) Y_{H} x^{*}$, where it is clear that $\lim _{Y_{H} \rightarrow 1} x^{*}=0 .{ }^{3}$ This shows the existence of an intermediate value of $Y_{H}$ maximizing $P_{H}^{*}$. Now, for each value of $Y_{H} \in[0,1)$ we compute the maximal productivity with respect to the dilution rate i.e. $\max \left\{P ; 0<D \leq\left(1-Y_{H}\right) \mu\left(S_{i n}, 0\right)\right\}$. These results are depicted in Figure 4. We observe that the optimal value of $Y_{H}$ is 0.505 , suggesting that protein productivity is maximal $\left(0.373 \mathrm{gL}^{-1} \mathrm{~d}^{-1}\right)$ when $50 \%$ of the absorbed substrate, that is not excreted in form of acetate, is diverted into protein production.

\footnotetext{
${ }^{2}$ Set $\mu^{\prime}(\cdot)=\left(1-Y_{H}\right) \mu(\cdot)$, and note that $\mu^{\prime}(\cdot)=Y_{S}^{\prime} r_{S}(\cdot)-Y_{R}^{\prime} r_{o f}(\cdot)$ with $Y_{S}^{\prime}=\left(1-Y_{H}\right) Y_{S}$ and $Y_{R}^{\prime}=\left(1-Y_{H}\right) Y_{R}$. Thus, Theorem 4.6 applies directly to $(32)$ when replacing $\left(1-Y_{H}\right) \mu(\cdot)$ by $\mu^{\prime}(\cdot)$.

${ }^{3}$ From (32), the intuition says that $x^{*}$ approaches 0 as $Y_{H}$ approaches 1 . This can be proved determining explicitly $x^{*}$ when $D \leq Y_{S}\left(1-Y_{H}\right)$ and using the upper bound for $x^{*}$ given in the proof of Proposition 3.1 when $D>Y_{S}\left(1-Y_{H}\right)$ (last paragraph).
} 


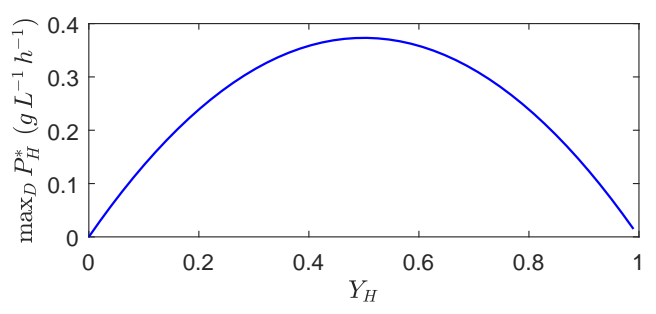

Figure 4: Maximal (with respect to $D$ ) protein productivity as a function of $Y_{H}$. The function $r_{S}$ is taken as in (2) and the parameters are taken from Table 1.

\section{Acknowledgements}

This work was supported by the INRIA project lab IPL-COSY and by the ANR project Maximic (ANR-11-LABX-0028-01).

\section{Appendix}

We present two results on differential inequalities. We only give the proof of the first result since both proofs are very similar. Consider the following differential equation:

$$
\frac{d y}{d t}=f(y, u) y
$$

with $f(y, u): \mathbb{R}_{+}^{2} \rightarrow \mathbb{R}$ a continuous function such that (34) admits a unique solution for all $t, u \in \mathbb{R}_{+}$and any initial condition $y(0)=y_{0} \geq 0$.

Lemma 5.1. Consider (34) and let $x: \mathbb{R}_{+} \rightarrow \mathbb{R}_{+}$be a function satisfying

$$
\frac{d x(t)}{d t} \leq f(x(t), u) x(t),
$$

for all $t, u \in \mathbb{R}_{+}$. Assume that $f(y, u)$ is strictly decreasing in $y$ and strictly increasing in $u$. Moreover, assume that $f(0,0)>0$ and that for any $u \in \mathbb{R}_{+}$ there is $M=M(u)>0$ such that $f(M, u)<0$. Then there is a unique $x^{*}>0$ such that $f\left(x^{*}, 0\right)=0$, and for any $\epsilon>0$ there is $u_{\epsilon}$ such that $x(t)-x^{*}<\epsilon$ for all $t \geq 0$ provided $x(0)-x^{*}<\epsilon / 2$ and $u<u_{\epsilon}$.

Proof. For any $u \geq 0$ we have $f(0, u) \geq f(0,0)>0$ and $f(M, u)<0$. Then, for any $u \geq 0$ there is a positive real number $a=a(u)$ such that $f(a(u), u)=0$. This proves the existence of $x^{*}:=a(0)$. Now note that $\lim _{u \rightarrow 0^{+}} a(u)=x^{*}$ and that $a(u)$ increases with respect to $u$. This implies that for a given $\epsilon>0$, there is $u_{\epsilon}>0$ such that $a\left(u_{\epsilon}\right)<x^{*}+\epsilon / 2$. Let $\bar{y}(t)$ be the solution to

$$
\frac{d y}{d t}=f\left(y, u_{\epsilon}\right)
$$


with $\bar{y}(0)=a\left(u_{\epsilon}\right)+\epsilon / 2$. Since (36) is an autonomous differential equation, $a\left(u_{\epsilon}\right)$ is the unique positive equilibrium of $(36)$, and $f\left(\bar{y}(0), u_{\epsilon}\right)<0$ we conclude that $\bar{y}(t) \leq a\left(u_{\epsilon}\right)+\epsilon / 2<x^{*}+\epsilon$ for all $t \geq 0$. Now, if $x(0)<x^{*}+\epsilon / 2$, then $x(0)<a\left(u_{\epsilon}\right)+\epsilon / 2=\bar{x}(0)$. In view of (37) Applying Theorem B1 in [28], we conclude that $x(t)<\bar{x}(t)<x^{*}+\epsilon$ for all $t \geq 0$.

Lemma 5.2. Consider (34) and let $x: \mathbb{R}_{+} \rightarrow \mathbb{R}_{+}$be a function -satisfying

$$
\frac{d x(t)}{d t} \geq f(x(t), u) x(t)
$$

for all $t, u \in \mathbb{R}_{+}$. Assume that $f(y, u)$ is strictly decreasing in both, $y$ and $u$. Moreover, assume that $f(0,0)>0$ and that for any $u \in \mathbb{R}_{+}$there is $M=$ $M(u)>0$ such that $f(M, u)<0$. Then there is a unique $x^{*}>0$ such that $f\left(x^{*}, 0\right)=0$ and for any $\epsilon>0$ there is $u_{\epsilon}$ such that $x^{*}-x(t)<\epsilon$ for all $t \geq 0$ provided $x^{*}-x(0)<\epsilon / 2$ and $u<u_{\epsilon}$.

\section{References}

[1] Abdelhamid Ajbar and Khalid Alhumaizi. Dynamics of the chemostat: A bifurcation theory approach. CRC Press, 2011.

[2] KR Babu, S Swaminathan, S Marten, N Khanna, and U Rinas. Production of interferon- $\alpha$ in high cell density cultures of recombinant escherichia coli and its single step purification from refolded inclusion body proteins. Applied microbiology and biotechnology, 53(6):655-660, 2000.

[3] Mohammed N Baeshen, Ahmed M Al-Hejin, Roop S Bora, MM Ahmed, HA Ramadan, Kulvinder S Saini, Nabih A Baeshen, and Elrashdy M Redwan. Production of biopharmaceuticals in e. coli: current scenario and future perspectives. J Microbiol Biotechnol, 25(7):953-962, 2015.

[4] Markus Basan, Sheng Hui, Hiroyuki Okano, Zhongge Zhang, Yang Shen, James R Williamson, and Terence Hwa. Overflow metabolism in escherichia coli results from efficient proteome allocation. Nature, 528(7580):99, 2015.

[5] Hans C Bernstein, Steven D Paulson, and Ross P Carlson. Synthetic escherichia coli consortia engineered for syntrophy demonstrate enhanced biomass productivity. Journal of biotechnology, 157(1):159-166, 2012.

[6] Anat Bren, Junyoung O Park, Benjamin D Towbin, Erez Dekel, Joshua D Rabinowitz, and Uri Alon. Glucose becomes one of the worst carbon sources for e. coli on poor nitrogen sources due to suboptimal levels of camp. Scientific reports, 6:24834, 2016.

[7] William Andrew Coppel. Stability and asymptotic behavior of differential equations. Heath, 1965. 
[8] Sofia Dashko, Nerve Zhou, Concetta Compagno, and Jure Piškur. Why, when, and how did yeast evolve alcoholic fermentation? FEMS yeast research, 14(6):826-832, 2014.

[9] Mark A Eiteman and Elliot Altman. Overcoming acetate in escherichia coli recombinant protein fermentations. Trends in biotechnology, 24(11):530$536,2006$.

[10] EMT El-Mansi and WH Holms. Control of carbon flux to acetate excretion during growth of escherichia coli in batch and continuous cultures. Microbiology, 135(11):2875-2883, 1989.

[11] Juana M Gancedo. Yeast carbon catabolite repression. Microbiol. Mol. Biol. Rev., 62(2):334-361, 1998.

[12] Luca Gerosa, Bart RB Haverkorn van Rijsewijk, Dimitris Christodoulou, Karl Kochanowski, Thomas SB Schmidt, Elad Noor, and Uwe Sauer. Pseudo-transition analysis identifies the key regulators of dynamic metabolic adaptations from steady-state data. Cell systems, 1(4):270-282, 2015.

[13] Jack K Hale. Asymptotic behavior of dissipative systems. Number 25. American Mathematical Soc., 2010.

[14] Emily Harvey, Jeffrey Heys, and Tomáš Gedeon. Quantifying the effects of the division of labor in metabolic pathways. Journal of theoretical biology, 360:222-242, 2014.

[15] MCM Hensing, RJ Rouwenhorst, JJ Heijnen, JP Van Dijken, and JT Pronk. Physiological and technological aspects of large-scale heterologous-protein production with yeasts. Antonie van Leeuwenhoek, 67(3):261-279, 1995.

[16] Julia Heßeler, Julia K Schmidt, Udo Reichl, and Dietrich Flockerzi. Coexistence in the chemostat as a result of metabolic by-products. Journal of mathematical biology, 53(4):556-584, 2006.

[17] Chung-Jr Huang, Henry Lin, and Xiaoming Yang. Industrial production of recombinant therapeutics in escherichia coli and its recent advancements. Journal of industrial microbiology $\& 3$ biotechnology, 39(3):383-399, 2012.

[18] Jung-whan Kim and Chi V Dang. Cancer's molecular sweet tooth and the warburg effect. Cancer research, 66(18):8927-8930, 2006.

[19] DJ Korz, U Rinas, K Hellmuth, EA Sanders, and W-D Deckwer. Simple fed-batch technique for high cell density cultivation of escherichia coli. Journal of biotechnology, 39(1):59-65, 1995. 
[20] Yuan-Shuai Liu and Jian-Yong Wu. Modeling of xanthophyllomyces dendrorhous growth on glucose and overflow metabolism in batch and fedbatch cultures for astaxanthin production. Biotechnology and bioengineering, 101(5):996-1004, 2008.

[21] Gregory W Luli and WILLIAM R Strohl. Comparison of growth, acetate production, and acetate inhibition of escherichia coli strains in batch and fed-batch fermentations. Appl. Environ. Microbiol., 56(4):1004-1011, 1990.

[22] Marco Mauri, Jean-Luc Gouzé, Hidde De Jong, and Eugenio Cinquemani. Enhanced production of heterologous proteins by a synthetic microbial community: Conditions and trade-offs. PLOS Computational Biology, 16(4):e1007795, 2020.

[23] Jacques Monod. La technique de culture continue: theorie et applications. 1950.

[24] K Nakano, M Rischke, S Sato, and H Märkl. Influence of acetic acid on the growth of escherichia coli k12 during high-cell-density cultivation in a dialysis reactor. Applied microbiology and biotechnology, 48(5):597-601, 1997.

[25] Aaron Novick and Leo Szilard. Description of the chemostat. Science, 112(2920):715-716, 1950.

[26] Lawrence Perko. Differential equations and dynamical systems, volume 7. Springer Science \& Business Media, 2013.

[27] Hal L Smith. Monotone Dynamical Systems: An Introduction to the Theory of Competitive and Cooperative Systems. Number 41. American Mathematical Soc., 2008.

[28] Hal L Smith and Paul Waltman. The theory of the chemostat: dynamics of microbial competition, volume 13. Cambridge university press, 1995.

[29] Horst R Thieme. Convergence results and a Poincaré-Bendixson trichotomy for asymptotically autonomous differential equations. Journal of Mathematical Biology, 30(7):755-763, 1992.

[30] Alexei Vazquez. Overflow metabolism: from yeast to marathon runners. Academic Press, 2017.

[31] Alan J Wolfe. The acetate switch. Microbiol. Mol. Biol. Rev., 69(1):12-50, 2005.

[32] Zhi-Long Xiu, An-Ping Zeng, and Wolf-Dieter Deckwer. Multiplicity and stability analysis of microorganisms in continuous culture: effects of metabolic overflow and growth inhibition. Biotechnology and bioengineering, 57(3):251-261, 1998. 
[33] Toshimasa Yano and Shozo Koga. Dynamic behavior of the chemostat subject to product inhibition. The Journal of General and Applied Microbiology, 19(2):97-114, 1973. 